\title{
The Complex Trajectory Method and Dissipation in Fission*
}

\author{
F. F. Karpeshin ** \\ Fock Institute of Physics, St. Petersburg University, St. Petersburg, 198504 Russia, \\ and Universidade de Coimbra, Departamento de Física, Coimbra, Portugal \\ Received August 28, 2002
}

\begin{abstract}
The sensitivity of the muon attachment probabilities to the light fragments of prompt fission $W_{l}$ on the fission dynamics is discussed. It is shown that the final $W_{l}$ value consists of two contributions: one from the point of scission and a second from avoided crossing of the levels. The relative contribution from the prescission phase turns out to be higher for more asymmetric fission. (C) $2003 \mathrm{MAIK}$ "Nauka/Interperiodica”.
\end{abstract}

\section{DISSIPATION IN FISSION}

A feature of fundamental interest of nuclear matter is dissipation of the collective energy. This feature is manifested in nuclear fission. From the experimental viewpoint, the dissipation is studied in the fusion-fission reaction, where emitted prefission gammas and neutrons and, sometimes, light charged particles play a role of a natural time scale.

Another situation is in low-energy fission, e.g., induced by thermal neutrons. In this case, no prefission gammas or neutrons have been positively observed. It is known that neutrons are emitted from fully accelerated fragments. And it can be concluded from this fact that prefission time $t_{\mathrm{sc}} \lesssim 10^{-19} \mathrm{~s}[1]$. This upper limit still remains two orders of magnitude higher than the concluded duration of around a few units of $10^{-21} \mathrm{~s}$ for the saddle-to-scission descent found in the case of heavy-ion collisions [2].

Some hopes had been related to ternary fission, accompanied by emission of light charged particles, but they were also not quite realized [3].

Usually, one considers two mechanisms of dissipation: one-body dissipation and two-body viscosity. The former comes from collisions of the nucleons with the moving walls of the fissile nucleus. The other is due to collisions between the nucleons. The onebody mechanism is generally characterized with a more compact scission configuration and, as a consequence, with less primary kinetic energy of the nascent fragments, smaller deformation energy, and larger Coulomb potential energy (Fig. 1). At first sight, all these features could be immediately revealed in the total kinetic energy (TKE) of the fragments,

\footnotetext{
*This article was submitted by the author in English.

** e-mail: karpesh@snoopy .phys.spbu.ru
}

their excitation energy, and the number of emitted neutrons and the radiated energy leaving with gamma quanta. However, all these three characteristic quantities are not independent, being mutually related by the conservation of energy. Thus, less primary kinetic energy is compensated by larger Coulomb potential energy, so that at infinity the TKE becomes the same for both scission configurations. Analogously, smaller deformation energy is balanced by higher intrinsic energy (that is, higher temperature) at the scission point. Therefore, measuring the TKE provides us with limited information about the scission configuration of the fragments. Furthermore, measuring the energy carried off by the neutrons, gammas, or light charged particles, in correlation with the TKE of the same fragments, provides the most of any conceivable information about the scission characteristics. However, again, only the sum of the deformation plus intrinsic energy of the fragment energy can be extracted in this way-in view of the conservation of their total value. There always remains a corridor of uncertainty for the $R_{\mathrm{sc}}$ value. An independent source of information is therefore needed if one wants to know the scission configuration more precisely. Muon-induced prompt fission can serve as a tool capable of providing unique information about the scission configuration versus its TKE.

Prompt fission occurs as a result of a radiationless transition to the $1 s$ state in a muonic atom [4-7]. Its study also offers a way of probing the fission dynamics.

As a result of prompt fission, the muon is usually entrained on the heavy fragment, forming a muonic atom. This gives rise to different processes and effects, such as muonic conversion of the $\gamma$ rays of the fragment, muon capture by the fragment, muonic $\mathrm{x}$-ray of the fragment, augmentation of the fission barrier due to the presence of the muon, and many 